UA-NPPS 01/12

November 2001

\title{
CRITICAL FLUCTUATIONS AT RHIC ${ }^{a}$
}

\author{
N. G. ANTONIOU, F. K. DIAKONOS and A. S. KAPOYANNIS \\ Department of Physics, University of Athens, GR-15771, \\ Athens, Greece
}

\begin{abstract}
On the basis of universal scaling properties, we claim that in $A u+A u$ collisions at RHIC, the QCD critical point is within reach. The signal turns out to be an extended plateau of net baryons in rapidity with approximate height $n_{b} \approx 15$ and a strong intermittency pattern with index $s_{2}=1 / 6$ in rapidity fluctuations. A window also exists, to reach the critical point at the SPS, especially in $\mathrm{Si}+\mathrm{Si}$ collisions at maximal energy.
\end{abstract}

\section{Introduction}

The existence of a critical point in the phase diagram of QCD is a fundamental property of strong interactions and therefore its experimental verification is of great importance. It is the remnant of a tricritical point, associated with the chiral phase transition, located at the end of a critical line of first order in the phase diagraml. Theoretical efforts to reveal the existence and location of the critical point, from first principles, are now in progress, in studies based on the Lee-Yang theory of phase transitions, treated on the lattice 2. On the other hand, the phenomenology of the QCD critical point in heavy-ion physics 13 becomes more and more promising due to the accumulation of measurements both from SPS and recently from RHIC. In this presentation we explore the scaling properties associated with the universality class of the QCD critical point in order (a) to derive quantitative criteria for a given experiment to drive the system close to the critical point and (b) to construct a theory of critical fluctuations in the net-baryon sector which can be tested in these experiments. On the basis of our predictions, a set of necessary and sufficient conditions is proposed, as a sharp signature of the QCD critical point at RHIC.

${ }^{a}$ Presented at the XXXI International Symposium on Multiparticle Dynamics, September 1-7, 2001, Datong, China 


\section{Net-baryon scaling}

The natural order parameter, incorporating the universal behaviour of quark matter near the critical point, is given by the classical behaviour of an isoscalar field ( $\sigma$-field) in $3 d$ with zero mass at the critical point. The fluctuations of the $\sigma$-field manifest themselves as density fluctuations in the pion sector and can be detected in heavy-ion experiments, especially in low multiplicity events 3 . Fluctuations of the same origin are expected to appear also in the net-baryon sector since near the critical point $\left(\rho_{c}, T_{c}\right)$ an equivalent order parameter is given by the difference of the net-baryon density $(\rho)$ from its critical value, $\langle\bar{q} q\rangle \sim\left(\rho-\rho_{c}\right)$. This new order parameter obeys the same scaling laws as the sigma-field, dictated by the critical exponents of the $3 d$ Ising system $(\beta \approx$ $\left.\frac{1}{3}, \delta \approx 5, \nu \approx \frac{2}{3}, \eta \approx 0\right)$. Approaching the critical point $\left(T \rightarrow T_{c}\right)$, the order parameter $m(\vec{x})=\rho-\rho_{c}$ satisfies, along the rapidity axis, a scaling law of the general form $\theta$ :

$$
\begin{aligned}
& m(y) \approx t^{\beta}\left[F_{o}(y / L)+t F_{1}(y / L)\right] ; \\
& m(y)=A_{\perp}^{-2 / 3} n_{b}(y)-\rho_{c}
\end{aligned}
$$

where $t \equiv \frac{T_{c}-T}{T_{c}}\left(T \leq T_{c}\right)$ and terms of order $O\left(t^{\beta+2}\right)$ have been neglected. Eq.(11) describes the critical behaviour of the net-baryon fluid, confined along the rapidity axis in the region $0 \leq y \leq L$ and extended in a large area of radius $R_{\perp} \sim A_{\perp}^{1 / 3}$ in the transverse space. The order parameter $m(y)$ is associated with the net-baryon rapidity density $n_{b}(y)$ produced in a heavy-ion collision $(A+A)$. If $A_{t}$ is the total number of participants in such a collision, equation (1) leads to the following scaling law in the neighbourhood of the critical point, along the freeze-out line of the process

$A_{\perp}^{-2 / 3} n_{b}(0)=\rho_{c}+\frac{4^{\beta / \nu}}{B\left(1-\frac{\beta}{\nu}, 1-\frac{\beta}{\nu}\right)}\left[f\left(z_{c}, \rho_{c}\right)\right]^{\beta}+C\left[f\left(z_{c}, \rho_{c}\right)\right]^{\beta+1} \quad ; \quad z_{c} \geq \rho_{c}$

$A_{\perp}^{-2 / 3} n_{b}(0) \approx z_{c} \quad ; \quad z_{c}<\rho_{c}$

The scaling variable in eq. (2) is $z_{c}=A_{\perp}^{-2 / 3} A_{t} L^{-1}\left(A_{\perp} \approx \frac{A_{t}}{2}\right)$ and the scaling function $f\left(z_{c}, \rho_{c}\right)$ vanishes for $z_{c}=\rho_{c}: f\left(z_{c}, \rho_{c}\right)=\frac{1}{G}\left(-1+\sqrt{1+2 G\left(z_{c}-\rho_{c}\right)^{1 / \beta}}\right)$. The constants $C$ and $G$ give a measure of the non leading term $F_{1}$ in eq.(1D) $\left(C \sim F(1 / 2), G \sim \int_{0}^{1} F_{1}(x) d x\right)$ and they are fixed by the measurements of net baryons at the SPS. In fact the trend of the data suggests the existence of a critical point $\left(\rho_{c} \approx 0.8\right)$ near the chemical potential of $S+S$ central collisions (Fig. 1).

The condition for a given experiment to reach the critical point, turns out to be the development of an extended plateau of net baryons in rapidity with 
height $n_{b} \approx 0.13 L^{2}$. At RHIC, this condition gives $n_{b} \approx 15$ and $A_{t} \approx 165$ whereas at the SPS the corresponding values are smaller, $n_{b} \approx 5$ and $A_{t} \approx 30$. As a result, the critical point may become accessible at RHIC with $A u+A u$ collisions whereas at the SPS it can only be reached in collisions of medium-size nuclei $(S+S, S i+S i)$.

\section{Critical fluctuations}

The net-baryon system, produced in a particular class of experiments with heavy ions, develops strong critical fluctuations in rapidity density if the constraints, discussed in the previous section, are satisfied. The origin of these fluctuations can betraced in the free energy of the $\sigma$-field at $T=T_{c}$, given by the effective action $B$ :

$$
\Gamma_{c}=\frac{\pi R_{\perp}^{2} \beta_{c}}{\tau_{c}} \int_{\delta y} d y\left[\frac{1}{2}\left(\frac{\partial \sigma}{\partial y}\right)^{2}+2 \tau_{c}^{2} \beta_{c}^{-4}\left(\beta_{c} \sigma\right)^{\delta+1}\right] \quad ; \quad \beta_{c} \equiv T_{c}^{-1}
$$

where $\delta$ is the appropriate critical exponent at $T=T_{c}(\delta \approx 5)$ and $\delta y$ the size of a critical cluster. The manifestation of $\sigma$-fluctuations in phase space, at the level of multiparticle production, is expected both in the pion sector $\left(\sigma \rightarrow \pi^{+} \pi^{-}\right)$and the net-baryon sector. At RHIC, the detection of fluctuations in the pion sector is not easy because the multiplicity in central collisions is high and the excess of pions, coming from sigmas, forms a subsystem of rather low multiplicity $\left(n_{c h}^{(\sigma)} \approx 150\right)$ in each event $\mathrm{B}$. Therefore the measurement of net-baryon fluctuations becomes a superior proposal for RHIC, in the search for the QCD critical point. In order to reveal the nature of the critical fluctuations in the net-baryon sector, we introduce the order parameter $m(y)$ through the equations:

$$
\begin{gathered}
\sigma(y) \approx F \beta_{c}^{2} m(y) ; F \equiv-\frac{\lambda\langle\bar{q} q\rangle_{o}}{\rho_{c}} ;\langle\bar{q} q\rangle_{o} \approx-3 f m^{-3} \\
\Gamma_{c} \approx g_{1} \int_{\delta y} d y\left[\frac{1}{2}\left(\frac{\partial \hat{m}}{\partial y}\right)^{2}+g_{2}|\hat{m}|^{\delta+1}\right] ; \hat{m}(y)=\beta_{c}^{3} m(y)
\end{gathered}
$$

where $g_{1} \equiv F^{2}\left(\frac{\pi R_{\perp}}{\tau_{c} \beta_{c}}\right), g_{2} \equiv 2 F^{4}\left(\frac{\tau_{c}}{\beta_{c}}\right)^{2}$. The partition function $Z=\int \mathcal{D}[\hat{m}] e^{-\Gamma_{c}[\hat{m}]}$ for each cluster is saturated by instanton-like configurations 目 which for $\delta y \leq \delta_{c}$ lead to self-similar structures characterized by a pair-correlation of the form:

$$
\langle\hat{m}(y) \hat{m}(0)\rangle \approx \frac{5}{6} \frac{\Gamma(1 / 3)}{\Gamma(1 / 6)}\left(\frac{\pi R_{\perp}^{2} \tau_{c}}{\beta_{c}^{3}}\right) F^{-1} y^{-\frac{1}{\delta+1}}
$$


The maximal size of these fractal clusters is $\delta_{c} \approx\left(\frac{\pi R_{\perp}^{2}}{16 \tau_{c}^{2}}\right)^{2 / 3}$, according to the geometrical description of a critical system $E$. The dimensionless parameter $F$ is of the order $10^{2}$ and the size $\delta_{c}$, on general grounds $\left(R_{\perp}<2 \tau_{c}\right)$, is of the order of one $\left(\delta_{c} \approx 0.35\right)$. As a result the global baryonic system (at RHIC the size of the system is $L \approx 11$ ) develops fluctuations at all scales in rapidity since the direct correlation (5) propagates along the entire system through the cooperation of many self-similar clusters of relatively small size. We have quantified this mechanism in a Monte-Carlo simulation for the conditions at RHIC, associated with the critical point $\left(L \approx 11, A_{t} \approx 165\right)$. In Fig. 2 a typical event of net baryonsat RHIC is presented, together with the corresponding intermittency pattern 6 revealing the nature of the critical fluctuations in rapidity. The intermittency exponent of the second moment $F_{2}\left(s_{2} \approx 0.18\right)$ is close to the value $d-d_{F}=1 / 6\left(d=1, d_{F}=\frac{\delta}{\delta+1}\right)$ expected from the universality class of the critical point. It is of interest to note that a stronger intermittency effect is expected in two and three dimensional events.

In Fig. 3 the inclusive distribution of net baryons is illustrated, built-up by $10^{3}$ underlying critical events. As expected, an extended plateau is generated in rapidity with height $n_{b} \approx 15$, in accordance with the diagram in Fig. 1 .

In conclusion, we have argued that a sharp signature of the QCD critical point at RHIC, consists of the following phenomena in the net-baryon sector:

- An extended plateau of net baryons in rapidity with height $n_{b} \approx 15$

- Strong intermittency fluctuations of net baryons in rapidity with index $s_{2} \approx 1 / 6$

The absence of these phenomena at RHIC $(A u+A u, \sqrt{s}=200 \mathrm{GeV})$ would indicate that, in these experiments, quark matter approaches the critical point in a state far from thermal equilibrium.

\section{References}

1. F. Wilczek, hep-ph/0003183; J. Berges and K. Rajagopal, Nucl. Phys. B 538, 215 (1999); M.A. Stephanov, K. Rajagopal and E. Shuryak, Phys. Rev. Lett. 81, 4816 (1998).

2. Z. Fodor and S.D. Katz, hep-lat/0106002.

3. N.G. Antoniou, Y.F. Contoyiannis, F.K. Diakonos, A.I. Karanikas and C.N. Ktorides, Nucl. Phys. A 693, 799 (2001).

4. N.G. Antoniou, Nucl. Phys. B 92, 26 (2001). 
5. N.G. Antoniou, Y.F. Contoyiannis, F.K. Diakonos and C.G. Papadopoulos, Phys. Rev. Lett. 81, 4289 (1998); N.G. Antoniou, Y.F. Contoyiannis and F.K. Diakonos, Phys. Rev. E 62, 3125 (2000).

6. A. Bialas and R. Peschanski, Nucl. Phys. B 273, 703 (1986); Nucl. Phys. B 308, 857 (1988); E.A. De Wolf, I.M. Dremin and W. Kittel, Phys. Rep. 270, 1 (1996). 


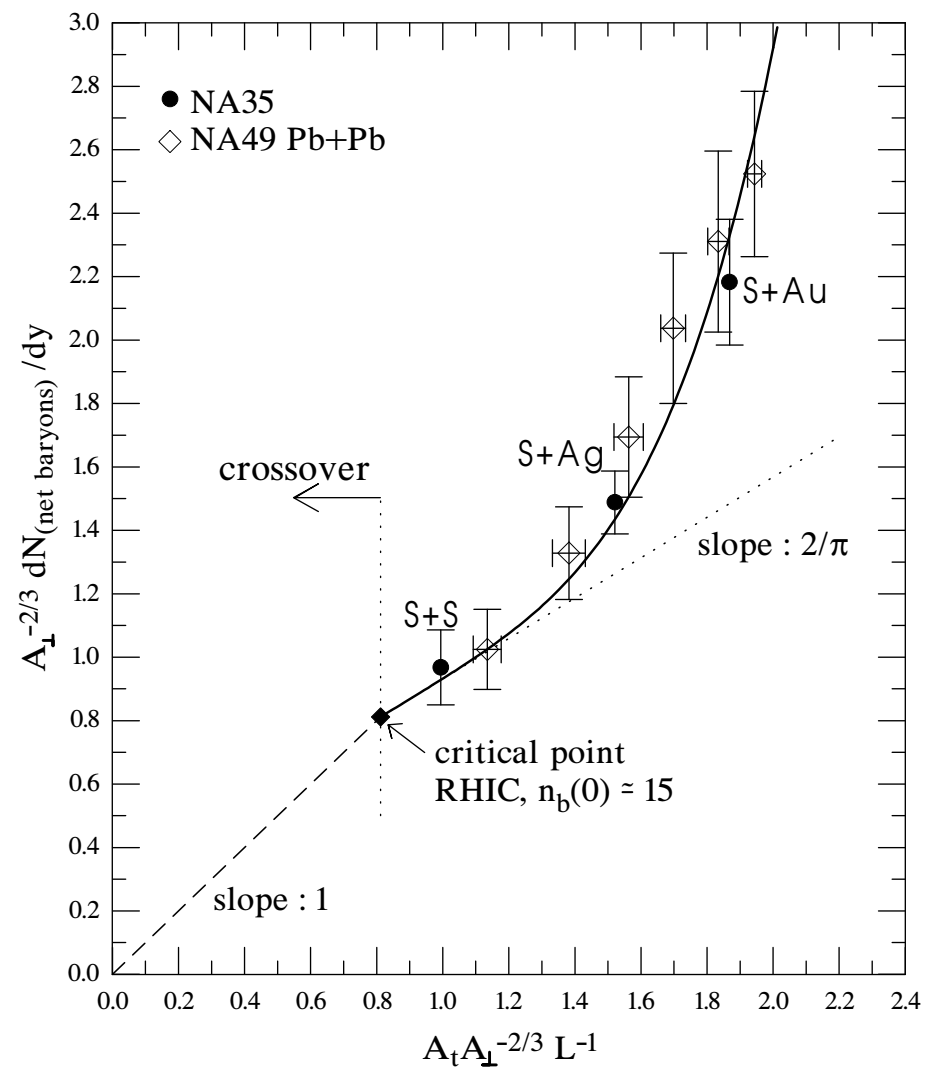

Figure 1: The diagram showing the proximity of heavy-ion experiments to the critical point. At RHIC, the critical point corresponds to the values $n_{b} \approx 15, A_{t} \approx 165$. 

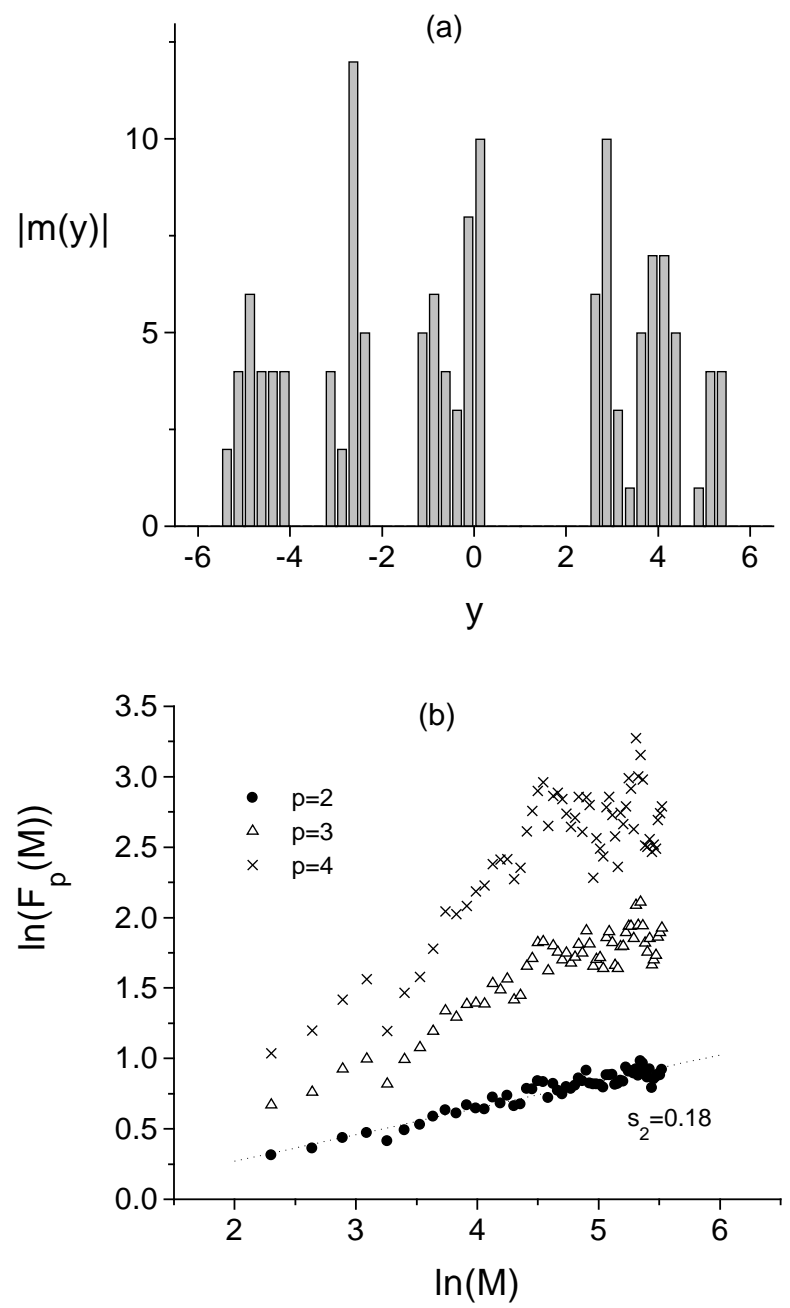

Figure 2: (a) The net-baryon fluctuations in rapidity space for a typical critical event. (b) The first three factorial moments for the net-baryon distribution in rapidity corresponding to the event shown in (a). 


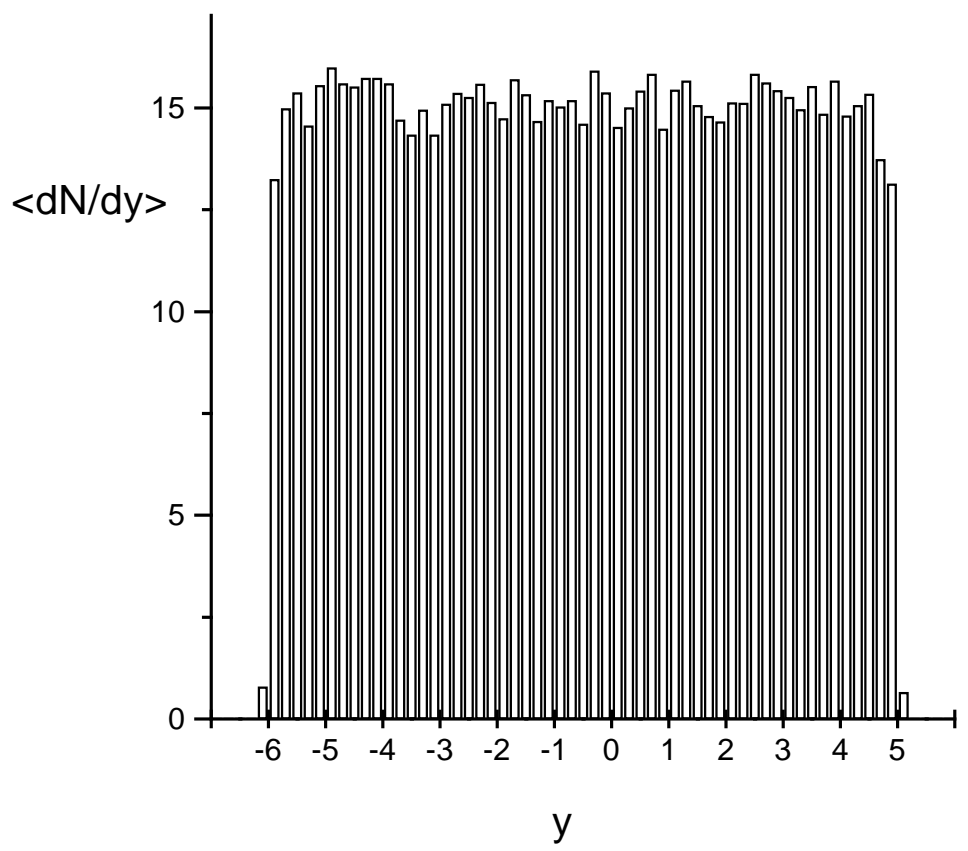

Figure 3: The inclusive net-baryon distribution in rapidity for $1000 \mathrm{MC}$ generated critical events. The parameters in the MC have been chosen in accordance with the conditions at RHIC. 